\title{
Test/Retest Reliability of Maximum Mouth Pressure Measurements With the MicroRPM in Healthy Volunteers
}

\author{
Zacharias Dimitriadis MSc, Eleni Kapreli PhD, Ioanna Konstantinidou, \\ Jacqueline Oldham $\mathrm{PhD}$, and Nikolaos Strimpakos $\mathrm{PhD}$
}

\begin{abstract}
BACKGROUND: Respiratory muscle strength is an important part of lung function. Assessment of the respiratory muscles' ability to generate force is important for recognizing respiratory muscle weakness in both sick and healthy people. OBJECTIVE: To assess the test/retest reliability of the MicroRPM portable manometer's measurements of maximum inspiratory pressure (MIP) and maximum expiratory pressure (MEP) in the sitting and standing positions; the number of expiratory maneuvers needed with the MicroRPM for reliability in MIP and MEP measurement; and the MicroRPM's test/retest reliability in other respiratory function indices, such as the maximum rate of pressure development (MRPD), the time constant of relaxation (tau), and the maximum relaxation rate (MRR). METHODS: We recruited 15 healthy volunteers (mean age 21.6 \pm 1.1 years). We assessed respiratory muscle strength on 3 separate occasions, each a week apart. We calculated reliability with the intraclass correlation coefficient (ICC), the standard error of measurement (SEM) and the smallest detectable difference (SDD). RESULTS: MicroRPM reliably measured MIP and MEP in both the sitting position (ICC 0.86-0.90, SEM 9-10, SDD 18-22) and standing position (ICC 0.78-0.83, SEM 12-14, SDD 23-26). After a 5-breath practice, 2 expiratory/inspiratory maneuvers on each testing occasion gave adequate MIP and MEP reliability (ICC $>0.90$ ). MRR reliability was moderate to excellent (ICC 0.58-0.87), MRPD reliability was moderate (ICC 0.59-0.64), and tau reliability was insufficient (ICC 0.27-0.67). CONCLUSIONS: The MicroRPM reliably measures MIP and MEP, but its MRPD, MRR, and tau measurements should be considered with caution. Key words: respiratory muscle; lung function; maximum expiratory pressure; maximum inspiratory pressure; manometer; reliability. [Respir Care 2011;56(6):776-782. @ 2011 Daedalus Enterprises]
\end{abstract}

\section{Introduction}

Respiratory muscle strength is an important part of lung function. ${ }^{1}$ Assessment of the respiratory muscles' ability

Mr Dimitriadis, Dr Kapreli, Ms Konstantinidou, and Dr Strimpakos are affiliated with the Physiotherapy Department, Technological Educational Institution of Lamia, Lamia, Greece. Mr Dimitriadis, Prof Oldham, and Dr Strimpakos are affiliated with the Centre for Rehabilitation Science, University of Manchester, Manchester, United Kingdom.

The authors have disclosed no conflicts of interest.

Correspondence: Eleni Kapreli PhD, Physiotherapy Department, Technological Educational Institution of Lamia, 3rd Km Old National Road, Lamia, Athens, 35100 Greece. E-mail: ekapreli@teilam.gr.

DOI: $10.4187 /$ respcare. 00783 to generate force is important for recognizing respiratory muscle weakness in both sick and healthy people. ${ }^{2}$ The importance of its assessment is represented by the fact that the findings from an appropriate evaluation of respiratory muscle strength may provide insight into unexplained dyspnea and insufficient cough. ${ }^{3}$ It is also useful as a complementary outcome measure for many disorders, ${ }^{4}$ such as neuromuscular disorders, ${ }^{5} \mathrm{COPD},{ }^{6}$ and heart failure. ${ }^{7}$ These reasons have strengthened the need to extensively research the development of reliable manometers for measuring respiratory muscle strength.

The literature describes many respiratory musclestrength-measurement tools, ${ }^{3,8}$ which can be divided into invasive and noninvasive. ${ }^{8}$ Although invasive techniques, such as esophageal and gastric balloons for recording esophageal, gastric, and transdiaphragmatic pressure, are considered more reliable, they require difficult, long, and un- 


\section{Test/Retest Reliability of Maximum Mouth Pressure Measurements With the MicroRPM}

pleasant procedures. ${ }^{3}$ Therefore, noninvasive procedures, such as measurement of mouth or nasal pressure, which are easily performed, are usually preferred and are widely applied and accepted, ${ }^{8}$ despite the fact that their results are effort-dependent, resulting in potentially less accurate values than non-volitional methods such as electrical stimulation, magnetic stimulation, or phonomyography. ${ }^{3,8}$

The maximum inspiratory pressure (MIP) and maximum expiratory pressure (MEP) reflect the respiratory muscles' ability to generate force during a short quasi-static contraction. ${ }^{9}$ MIP and MEP measurement is conducted with a manometer that measures mouth-pressure, and can be affected by lack of patient motivation or coordination. ${ }^{3}$ In addition to their noninvasive characteristics and the fact that normative MIP and MEP values have been established in many studies, ${ }^{10-13}$ the portability of a MIP/MEP manometer enables assessment both in the clinic and at the bedside. ${ }^{14,15}$

The MicroRPM (Micro Medical/CareFusion, Kent, United Kingdom) is a modern manometer that was recently used in studies that recorded MIP and MEP. ${ }^{16,17}$ Although maximum-mouth-pressure measurements with some portable manometers have been found reliable and valid both in healthy volunteers and pulmonary and neuromuscular patients, ${ }^{2,9,14,18-21}$ there is no published evidence about the reliability of the MicroRPM. Most previous manometer reliability studies used inappropriate or insufficient statistical indices of reliability (eg, Pearson correlation coefficient), making their assumptions problematic. $2,9,14,20$

Moreover, the measurement reliability of other respiratory-function indices that can be measured with the MicroRPM is unknown. These indices are the maximum rate of pressure development (MRPD, which is the initial linear incline of the maximum respiratory pressure curve, which reflects muscle function), the time constant of relaxation (tau), and the maximum relaxation rate (MRR). A slowing in the rate of decline, confirmed by an increase of tau or a slowing of MRR, reflects respiratory muscle fatigue. ${ }^{20}$

The main objectives of this study were to investigate the MicroRPM's test/retest reliability for measuring MIP and MEP in healthy people, in both the sitting and standing positions, and to determine the needed number of repetitions with each subject, in one session, to obtain reliable MIP and MEP values. We recruited healthy people to find the measurement error independent of any pathologies. A secondary objective was to examine the MicroRPM's test/ retest reliability in measuring MRR, MRPD, and tau. Test/ retest reliability is defined as the degree to which an instrument's measurements are stable when the measurement administered to the same individuals over a specified time period and the measurement conditions remain the same. ${ }^{22}$

\section{Methods}

This study was approved by the ethics committee of, and performed in the Physiotherapy Department of the Technological Educational Institution of Lamia, Lamia, Athens, Greece, and performed according to the Declaration of Helsinki. All participants gave written informed consent.

\section{Subjects}

We recruited a convenience sample of 15 healthy volunteers ( 9 males, 6 females) who were physiotherapy students of the Technological Educational Institution of Lamia. Participants were required to be between 18 and 50 years old and healthy to be included. The exclusion criteria were obesity, history of respiratory or neuromuscular disease, pulmonary infection, menstrual period, smoking, and drug consumption during the 2 weeks before the measurements, as these are known to influence respiratory function. To screen for study eligibility we used a demographics questionnaire, and a digital body fat caliper (FatTrack II, Accufitness, Greenwood Village, Colorado) for determining obesity.

\section{Protocol}

Demographic characteristics were recorded at baseline, followed by recording of MIP and MEP, in 3 testing sessions, a week apart from each other. The measurements took place at the same time of day for each participant. Volunteers were asked to perform 18 maximum inspiratory efforts and 18 maximum expiratory efforts in both the sitting and standing position, ${ }^{23,24}$ with a 30 -seconds interval between efforts, and 3-min interval between the 4 test conditions (sitting MIP, standing MIP, sitting MEP, and standing MEP). The tests were in random order. At all the testing sessions, instructions about the procedure were given in a standardized manner. All the measurements were performed by an appropriately trained physical therapist.

\section{Measurements}

MIP and MEP were measured with the MicroRPM, which is a small, portable, lightweight, noninvasive, mouth-pressure manometer with a rubber flanged mouthpiece and a small monitor that digitally displays the test results in $\mathrm{cm}_{2} \mathrm{O}$. The display uses a piezo resistive pressuresensing technology. The MicroRPM's Software (Puma PC, Micro Medical, Kent, England) calculates the MIP, MEP, MRPD, MRR, and tau. The MIP and MEP values are calculated from the one-second average maximum pressure. 


\section{Test/Retest Reliability of Maximum Mouth Pressure Measurements With the MicroRPM}

Before the measurements, each participant performed 5 maximum inspiratory and 5 maximum expiratory warm-up efforts to familiarize with the procedure. During the MIP and MEP measurement the participant was asked to hold the gauge with both hands and to close his or her lips firmly around the flanged mouthpiece. We applied a nose clip to avoid nasal air leak. For the MEP maneuver, the participant was asked to inhale as much as possible and then to exhale maximally for more than one second against the resistance of the gauge. For the MIP maneuver the participant was asked to exhale as much as possible (to residual volume) and then to inhale maximally for more than one second against the resistance of the gauge. ${ }^{4}$ All measurements were recorded for analysis.

\section{Data Analysis}

Data analysis was performed with statistics software (SPSS 15.0, SPSS, Chicago, Illinois). The normality of the data were evaluated with the Kolmogorov-Smirnov test. The test/retest reliability of all respiratory measurements was calculated with the intraclass correlation coefficient (ICC), the standard error of measurement (SEM, which is the square root of the within-subject mean squared error from the repeated-measures analysis of variance), and the smallest detectable difference (SDD, which is $1.96 \times \sqrt{2}$ $\times$ SEM, expressed as a percentage of the parameter's grand mean). ${ }^{22} \mathrm{We}$ calculated the inter-session test/retest reliability for all 3 sessions (all-3-inter-session ICC) and for the last 2 sessions (last-2-inter-session ICC). We calculated the intra-session ICC for both the mean MIP and MEP and the best MIP and MEP of the 18 repetitions. ICC values more than 0.6 were considered acceptable reliability, and ICC values more than 0.8 were considered high reliability. ${ }^{25}$ Sample size calculation revealed that the appropriate sample size for ICC values $\geq 0.9$ was 11 participants $\left(\mathrm{w}=0.2, \alpha=.05,3\right.$ measurements). ${ }^{26} \mathrm{We}$ recruited 15 participants to increase the power.

We used the Bland-Altman method, which includes a scatter plot of the differences between test and retest against their mean, to determine the magnitude of disagreement between the second and third sessions' values. ${ }^{27}$ This method also includes the $95 \%$ limits of agreement of differences and the mean difference. To evaluate changes over time in an individual (eg, the effect of clinical rehabilitation), the magnitude of the change has to exceed the inherent variability of the outcome. Thus, if the limits of agreement are very broad and clinically important, it suggests that test/retest could result in a clinically meaningful intra-patient difference.

We used the Spearman-Brown formula to estimate the number of repetitions (k) necessary to achieve an ICC $>0.9 .{ }^{28}$ We calculated the reliability coefficient of
Table 1. Subjects*

\begin{tabular}{lc}
\hline \hline & Mean $\pm \mathrm{SD}$ \\
\hline Age $(\mathrm{y})$ & $21.6 \pm 1.1$ \\
Height $(\mathrm{cm})$ & $173 \pm 8$ \\
Weight $(\mathrm{kg})$ & $68.3 \pm 11.6$ \\
Body mass index $\left(\mathrm{kg} / \mathrm{m}^{2}\right)$ & $20.3 \pm 3.5$ \\
& \\
\hline *9 male, 6 female & \\
\hline
\end{tabular}

the mean $\left(\mathrm{R}_{\mathrm{k}}\right)$ by averaging $\mathrm{k}$ repetitions with a one-measure reliability coefficient $(\mathrm{R})$ :

$$
\mathrm{R}_{\mathrm{k}}=\mathrm{kR} /[1+(\mathrm{k}-1) \mathrm{R}]
$$

We calculated the required number of repetitions (k) as:

$$
\mathrm{k}=[\mathrm{R} *(1-\mathrm{R})] /[\mathrm{R}(1-\mathrm{R} *)]
$$

where $\mathrm{R}^{*}$ is the target coefficient of reliability.

\section{Results}

Table 1 describes the subjects, who were young, thin, and with no mouth pathologies that could preclude maximum effort or mouth pressure.

The Kolmogorov-Smirnov test showed that all the data were normally distributed, so we used parametric statistics. Table 2 shows the inter-session ICC, SEM, and SDD values. The all-3-inter-session ICC range was $0.82-0.90$ for MEP, and 0.78-0.87 for MIP. MIP and MEP had higher ICC values in the sitting position (mean MEP all3-inter-session ICC 0.88, best MEP all-3-inter-session ICC 0.90, mean MIP all-3-inter-session ICC 0.86, best MIP all-3-inter-session ICC 0.87 ) than in standing position (mean MEP all-3-inter-session ICC 0.82, best MEP all-3inter-session ICC 0.83, mean MIP all-3-inter-session ICC 0.78 , best MIP all-3-inter-session ICC 0.81 ), but these reliability indices were very good for both positions.

The mean scores yielded similar ICC values (mean MEP all-3-inter-session ICC range 0.82-0.88, mean MIP all-3inter-session ICC range $0.78-0.86$ ) to the best scores (best MEP all-3-inter-session ICC range 0.83-0.90, best MIP all-3-inter-session ICC range $0.81-0.87$ ) in both positions.

All of these reliability indices were improved when the results from the first testing occasion were discarded. The SEM and SDD values were relatively small for all the variables. The Bland-Altman plots (Figs. 1 and 2) show that the limits of agreement were reasonably narrow, indicating small bias and systematic error. MRR had moderate to excellent reliability (all-3-inter-session ICC range 0.58-0.87), MRPD had moderate reliability (all-3-intersession ICC range 0.59-0.64), and tau had insufficient 


\section{Test/Retest Reliability of Maximum Mouth Pressure Measurements With the MicroRPM}

Table 2. Test/Retest Reliability

\begin{tabular}{|c|c|c|c|c|c|c|c|c|c|}
\hline & & \multicolumn{4}{|c|}{ All 3 Sessions } & \multicolumn{4}{|c|}{ Last 2 Sessions } \\
\hline & & Grand Mean & ICC & SEM & $\mathrm{SDD} \%$ & Grand Mean & ICC & SEM & SDD $\%$ \\
\hline \multirow[t]{2}{*}{ Mean MEP $\left(\mathrm{cm} \mathrm{H}_{2} \mathrm{O}\right)$} & Sitting & 136 & 0.88 & 9 & 19 & 139 & 0.89 & 10 & 20 \\
\hline & Standing & 136 & 0.82 & 12 & 25 & 139 & 0.89 & 10 & 20 \\
\hline \multirow[t]{2}{*}{ Best MEP $\left(\mathrm{cm} \mathrm{H}_{2} \mathrm{O}\right)$} & Sitting & 152 & 0.90 & 10 & 18 & 154 & 0.87 & 12 & 22 \\
\hline & Standing & 152 & 0.83 & 14 & 25 & 154 & 0.89 & 11 & 20 \\
\hline \multirow[t]{2}{*}{ Mean MIP $\left(\mathrm{cm} \mathrm{H}_{2} \mathrm{O}\right)$} & Sitting & 124 & 0.86 & 10 & 22 & 126 & 0.92 & 7 & 16 \\
\hline & Standing & 126 & 0.78 & 12 & 26 & 131 & 0.89 & 9 & 19 \\
\hline \multirow[t]{2}{*}{ Best MIP $\left(\mathrm{cm} \mathrm{H}_{2} \mathrm{O}\right)$} & Sitting & 140 & 0.87 & 10 & 21 & 141 & 0.90 & 9 & 18 \\
\hline & Standing & 140 & 0.81 & 12 & 23 & 145 & 0.94 & 8 & 14 \\
\hline \multirow[t]{2}{*}{ Inspiratory MRPD $\left(\mathrm{cm} \mathrm{H}_{2} \mathrm{O} / \mathrm{s}\right)$} & Sitting & 757 & 0.61 & 192 & 70 & 779 & 0.74 & 186 & 66 \\
\hline & Standing & 812 & 0.59 & 215 & 73 & 840 & 0.77 & 181 & 60 \\
\hline \multirow[t]{2}{*}{ Inspiratory MRR (Hz) } & Sitting & 3.6 & 0.87 & 0.6 & 46 & 3.7 & 0.86 & 0.7 & 52 \\
\hline & Standing & 3.5 & 0.78 & 0.8 & 66 & 3.6 & 0.79 & 0.8 & 62 \\
\hline \multirow[t]{2}{*}{ Inspiratory tau } & Sitting & -148 & 0.67 & 90 & 169 & -139 & 0.56 & 106 & 212 \\
\hline & Standing & -323 & 0.27 & 549 & 472 & -327 & 0.14 & 621 & 527 \\
\hline \multirow[t]{2}{*}{ Expiratory MRPD $\left(\mathrm{cm} \mathrm{H}_{2} \mathrm{O} / \mathrm{s}\right)$} & Sitting & 717 & 0.62 & 149 & 57 & 682 & 0.57 & 147 & 60 \\
\hline & Standing & 758 & 0.64 & 169 & 62 & 752 & 0.60 & 178 & 66 \\
\hline \multirow[t]{2}{*}{ Expiratory MRR (Hz) } & Sitting & 3.2 & 0.58 & 0.9 & 77 & 3.3 & 0.56 & 1.0 & 86 \\
\hline & Standing & 3.4 & 0.77 & 0.6 & 51 & 3.4 & 0.82 & 0.5 & 43 \\
\hline \multirow[t]{2}{*}{ Expiratory tau } & Sitting & -92 & 0.64 & 34 & 103 & -89 & 0.67 & 31 & 95 \\
\hline & Standing & -98 & 0.51 & 44 & 123 & -87 & 0.57 & 35 & 111 \\
\hline \multicolumn{10}{|l|}{$\begin{array}{l}\text { ICC }=\text { intraclass correlation coefficient } \\
\text { SEM = standard error of measurement } \\
\text { SDD } \% \text { smallest detectable difference as } \\
\text { MEP = maximum expiratory pressure } \\
\text { MIP = maximum inspiratory pressure } \\
\text { MRPD = maximum rate of pressure devel } \\
\text { MRR = maximum relaxation rate } \\
\text { tau = time constant of relaxation }\end{array}$} \\
\hline
\end{tabular}

reliability (all-3-inter-session ICC range 0.27-0.67). MRR, MRPD, and tau all had high SEM and SDD values, which makes their clinical application questionable.

Table 3 shows the intra-session ICC values, which were all very high (intra-session ICC range 0.87-0.91). Only 2 expiratory maneuvers and one standing inspiratory maneuver were needed for an ICC $>0.9$. Figure 3 shows the incremental curves of MIP and MEP reliability, calculated with the Spearman-Brown formula.

\section{Discussion}

This study provides new evidence regarding the reliability of the MicroRPM, suggesting that it can be used with high confidence in research and especially in clinical practice for the assessment of MIP and MEP. The ICC values we obtained for most of the MIP and MEP measurements were higher than 0.8 , indicating high reliability. ${ }^{25}$ Moreover, the low SEM and SDD values also confirm the usefulness of these measurements in clinical practice, as only a small amount of error needs to be overcome for detecting a real change. ${ }^{22}$ Discarding the first-session values increased the reliability further, which can be attributed to a partial elimination of learning effects. Interestingly, only 2 repetitions per session were needed to obtain reliable MIP and MEP measurements. Although the MIP and MEP measurements were equally reliable, the sitting position gave more reliable estimates than the standing position. However, the MRR, MRPD, and tau cannot be considered reliable.

Our MIP and MEP results agree with other reports. ${ }^{2,9,14,20,21}$ Larson and co-workers ${ }^{9}$ assessed the reliability of an aneroid manometer for measuring MIP in patients with COPD. Although that analysis was inappropriately performed with the Pearson correlation coefficient (r), MIP reliability was very high (r 0.97) when the MIP data were taken from the third and forth test sessions. Similar were the conclusions by Hamnegard and co-workers, ${ }^{14}$ who compared MIP and MEP measurements with a portable manometer versus a laboratory standard, in healthy people and patients with respiratory disease. Hamnegard et al concluded that the portable manometer was reliable and accurate for MIP and MEP, as the Bland-Altman plot revealed small bias and narrow limits of agreement. How- 
A
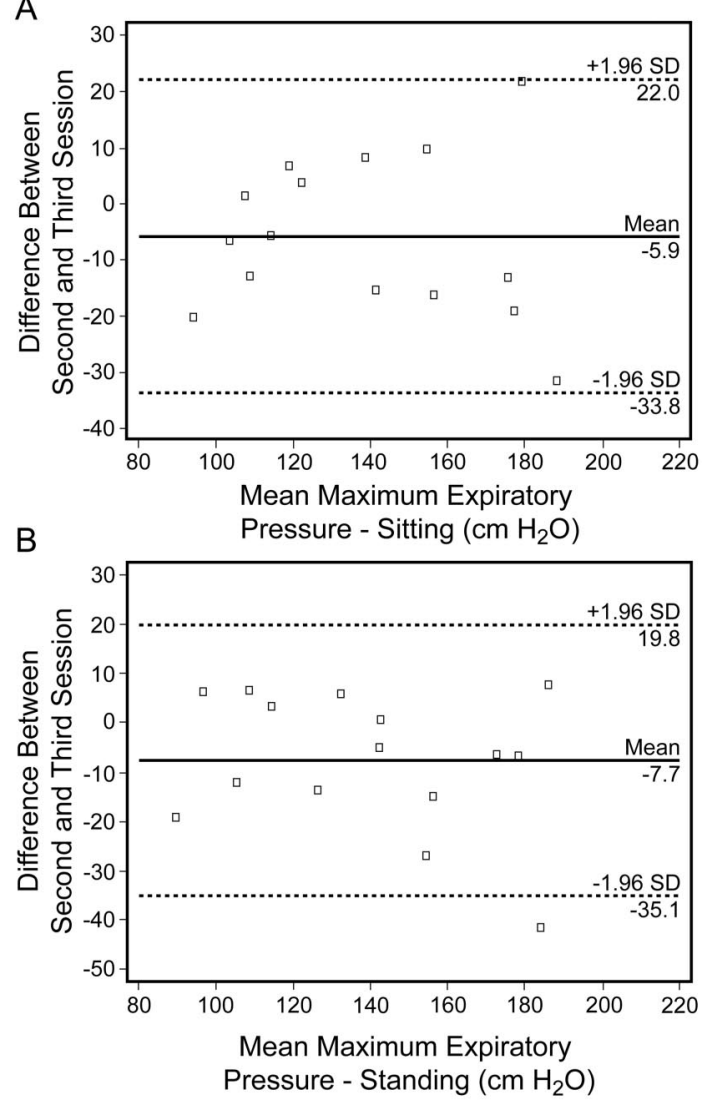

Fig. 1. Bland-Altman plot of intra-subject difference (test/retest difference) versus intra-subject mean (of 18 expiratory/inspiratory maneuvers) for maximum expiratory pressure in the sitting $(A)$ and standing (B) positions. The dashed lines indicate the $95 \%$ limits of agreement. The solid line represents the mean difference between sessions.

ever, their Bland-Altman plots compared the agreement between 2 sessions rather than the agreement of the portablemanometer measurements to those from the laboratory tool, which put more focus on reliability than on accuracy.

McConnell and Copestake ${ }^{2}$ examined the reliability of a hand-held manometer for measuring MIP and MEP in healthy elderly subjects, and constructed Bland-Altman plots similar to ours in the present study. The manometer they tested was very reliable: the agreement between the 2 sessions was $-0.3 \pm 6.4 \mathrm{~cm} \mathrm{H}_{2} \mathrm{O}$ for $\mathrm{MIP}$, and $1.4 \pm 5.1 \mathrm{~cm} \mathrm{H}_{2} \mathrm{O}$ for MEP. Those limits of agreement are better than we found in the present study, which might be explained by the different study populations. The elderly tend to have less respiratory muscle strength than healthy young adults. Our Bland-Altman plots show a slightly greater dispersion of MIP and MEP around the mean difference of the 2 sessions when MIP and MEP are high. This suggests that lower respiratory strength (such as in the elderly) might lead to less bias and narrower limits of agreement. On the other hand, although Romer and McConnell ${ }^{20}$ examined the reliability of a hand-held manom-
A
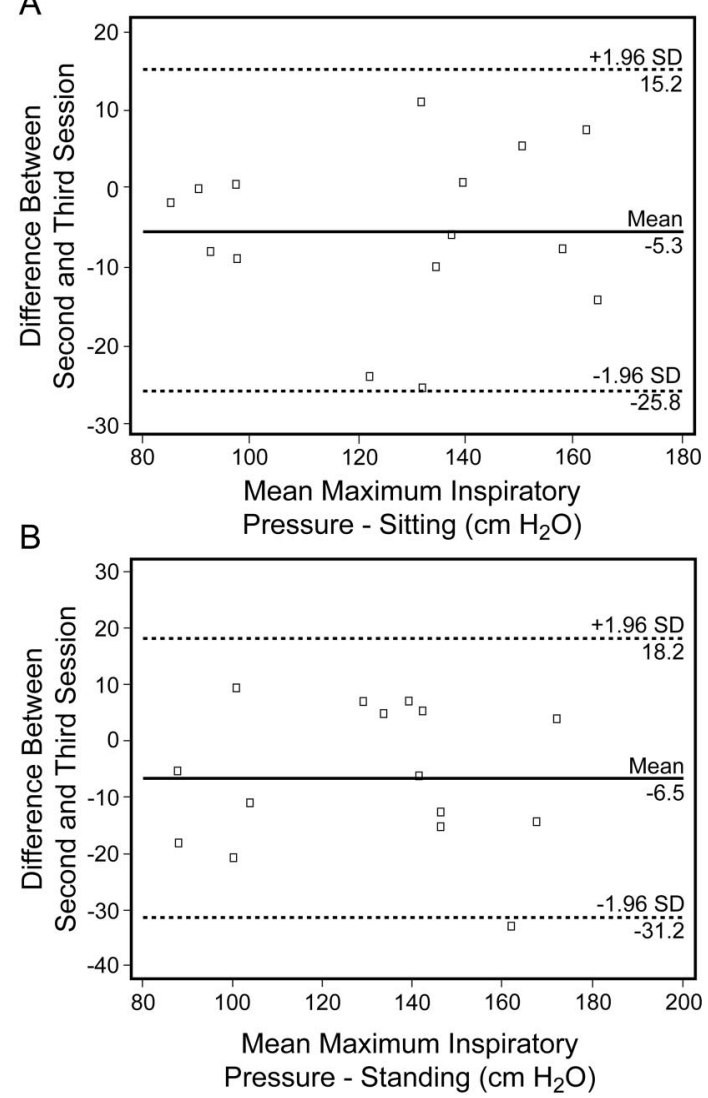

Fig. 2. Bland-Altman plot of intra-subject difference (test/retest difference) versus intra-subject mean (of 18 expiratory/inspiratory maneuvers) for maximum inspiratory pressure in the sitting $(A)$ and standing (B) positions. The dashed lines indicate the $95 \%$ limits of agreement. The solid line represents the mean difference between sessions.

eter for measuring MIP and MEP in a population quite similar to ours, they came to a similar conclusion, as their Bland-Altman plots showed negligible bias and narrow limits of agreement. However, in that study the interval between the 2 sessions ranged from 2 days to 3 weeks, which affects the standardization of the procedure and might explain their narrower limits of agreement, because the potential measurement of most of the participants during the first week may give less potential for historical effects. Finally, Maillard and co-workers ${ }^{21}$ reported high reliability (ICC $0.88-0.92$ ) of MIP in young healthy people, measured with a portable manometer. Those ICC values agree with our findings and indicate clinically acceptable reliability of these manometers.

Our MEP values are similar to those in other reports, considering sex, equipment (flanged mouthpiece), and protocol, whereas our MIP values are slightly higher. ${ }^{4,13} \mathrm{~A}$ possible reason is that we measured MIP starting at residual volume. Evans and Whitelaw, ${ }^{13}$ in their 2009 review, concluded also that MIP is approximately $16 \%$ larger at residual volume, in comparison with TLC. To establish 


\section{Test/Retest Reliability of Maximum Mouth Pressure Measurements With the MicroRPM}

Table 3. Intra-Session Reliability and Repetitions Required for Intraclass Correlation Coefficient $\geq 0.90$

\begin{tabular}{|c|c|c|c|c|c|}
\hline & Position & $\begin{array}{l}\text { Real Intra-Session } \\
\text { ICC (18 repetitions) }\end{array}$ & $95 \% \mathrm{CI}$ & $\begin{array}{l}\text { Real Intra-Session } \\
\text { ICC ( } 2 \text { repetitions) }\end{array}$ & $\begin{array}{l}\text { Repetitions Required } \\
\text { for ICC } \geq 0.90\end{array}$ \\
\hline \multirow[t]{2}{*}{ Maximum expiratory pressure } & Sitting & 0.87 & $0.78-0.95$ & 0.91 & 2 \\
\hline & Standing & 0.89 & $0.82-0.96$ & 0.92 & 2 \\
\hline \multirow[t]{2}{*}{ Maximum inspiratory pressure } & Sitting & 0.88 & $0.79-0.95$ & 0.95 & 2 \\
\hline & Standing & 0.91 & $0.84-0.96$ & 0.92 & 1 \\
\hline
\end{tabular}
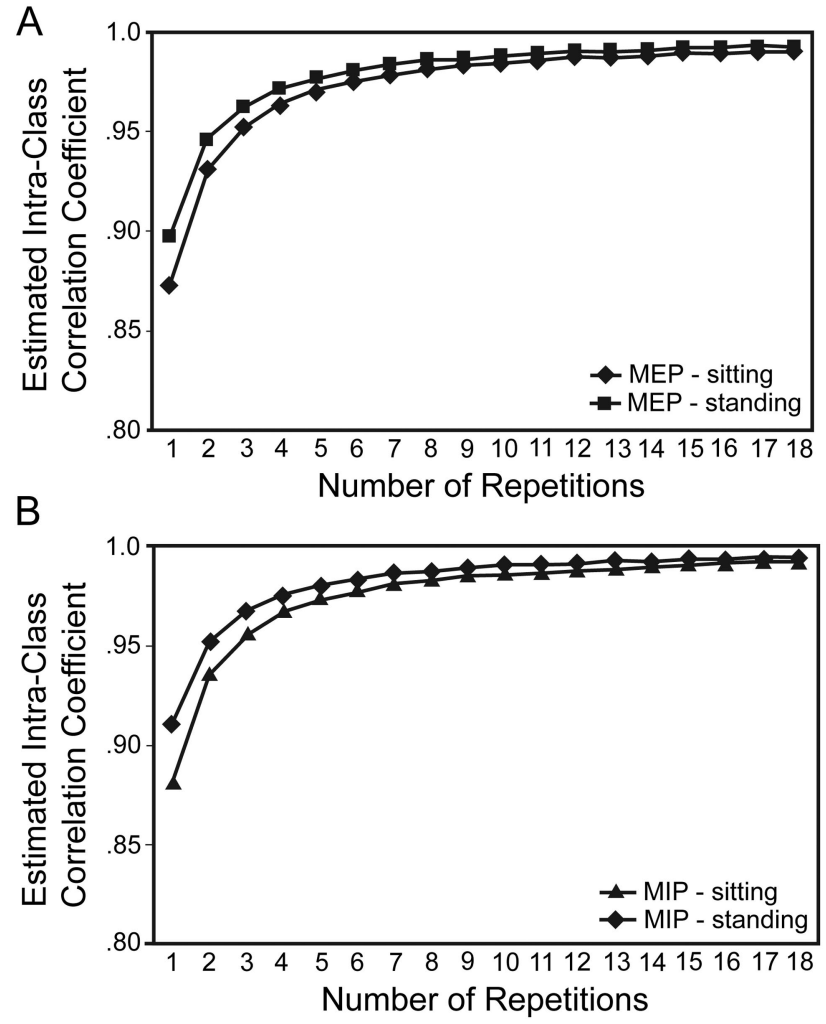

Fig. 3. Estimated intraclass correlation coefficients for repeated measurements of $(A)$ maximum expiratory pressure and $(B)$ maximum inspiratory pressure in the sitting and standing positions. The necessary number of repetitions is averaged to estimate the reliability.

MicroRPM's accuracy, however, requires a separate study of MicroRPM versus validated tools and techniques.

Another interesting finding of the present study is the similarity of MIP and MEP reliability in the standing and sitting positions, which were both highly reliable, especially when the first-session data were omitted. Thus, the standing or sitting position can be used with confidence, depending on the protocol under investigation.

Interestingly, unlike MIP and MEP, the MRPD, MRR, and tau were less reliable, which does not support their use in clinical research. However, these results disagree with the findings by Romer and McConnell. ${ }^{20}$ Their BlandAltman plots suggested that, in contrast to MRPD, the
MRR and tau had acceptable bias and limits of agreement. Although the discrepancy between these studies is difficult to explain, we think that reducing the time interval between the test sessions may give more consistent values. Therefore, the reliability of MRPD, MRR, and tau remain unclear and merit future study.

One of our main objectives was to determine the number of inspiratory and expiratory maneuver repetitions per session needed for reliable MIP and MEP, since there is no consensus in the literature. ${ }^{23,24}$ Our volunteers all performed 18 repetitions per session (the maximum suggested number of repetitions), which can be extremely time-consuming, especially since each effort has to be followed by an adequate recovery period to avoid fatigue. Moreover, factors such as fatigue (peripheral and central), learning effect, and inattention over time can all affect reliability estimates. This is obvious in Table 3, where the real ICC values were rarely higher than 0.90 , despite 18 repetitions (after 5 warm-ups), in contrast to the estimated ICC values obtained with the Spearman-Brown formula (see Fig. 3), where those factors could not be considered. On the other hand, our statistical analysis indicates that only 2 repetitions, average, are needed to obtain excellent reliability (ICC > 0.90) for both MIP and MEP. This conclusion is confirmed by both the predicted ICC values obtained with the Spearman-Brown formula and the real ICC values calculated from the first 2 repetitions (see Table 3). These results also agree with previous research ${ }^{24,29}$ and this finding emphasizes that, even without prior submaximal respiratory activity (as has been suggested), a well organized protocol including a familiarization procedure (ie, with 5 maximum efforts) can provide reliable MIP and MEP measurements with very few repetitions.

Our findings are very important for clinicians and researchers. Clinicians are provided with a reliable, noninvasive, and convenient MIP and MEP measurement tool that is acceptable to patients and can be used at the bedside, sitting or standing. The mean and maximum MIP and MEP values from 18 repetitions were equally reliable, and choosing the number of repetitions can be dependent on the rationale of the researcher and the purpose of the study. 


\section{Test/Retest Reliability of Maximum Mouth Pressure Measurements With the MicroRPM}

Our findings also suggest additional studies of the MicroRPM such as its inter-rater reliability and the validity of its measurements compared to well established invasive and noninvasive techniques. The MicroRPM should also be validated in various patient populations, such as patients with COPD, to examine its suitability to investigate and diagnose disease.

\section{Conclusions}

The MicroRPM reliably measured MIP and MEP in the sitting and standing positions. Following a 5-breath maximum-effort warm-up, 2 repetitions are needed to ensure MIP and MEP reliability. The MicroRPM's MRPD, MRR, and tau measurements should be interpreted with caution, as their reliability has not been confirmed. The establishment of MicroRPM's MIP and MEP reliability provides clinicians and researchers with a valid noninvasive measurement tool for respiratory muscle strength, which has high clinical utility.

\section{ACKNOWLEDGMENTS}

We thank Labrini Komianou PT for technical assistance, Vagellis Vourazanis PT for help during data selection, Ourania Kolokotroni MD for reviewing the manuscript, and Papathomas Michail MD, Department of Epidemiology and Public Health, Imperial College London, for assistance with statistical analysis.

\section{REFERENCES}

1. Kaminsky DA. Spirometry and related tests. In: Ruppel GL, editor. Manual of pulmonary function testing, 9 th edition. St Louis: Mosby; 2009:36-89.

2. McConnell AK, Copestake AJ. Maximum static respiratory pressures in healthy elderly men and women: issues of reproducibility and interpretation. Respiration 1999;66(3):251-258.

3. Syabbalo N. Assessment of respiratory muscle function and strength. Postgrad Med J 1998;74(870):208-215.

4. American Thoracic Society/European Respiratory Society. ATS/ERS statement on respiratory muscle testing. Am J Respir Crit Care Med 2002;166(4):518-624.

5. Mier-Jedrzejowicz A, Brophy C, Moxham J, Green M. Assessment of diaphragm weakness. Am Rev Respir Dis 1988;137(4):877-883.

6. Similowski T, Derenne JP. Inspiratory muscle testing in stable COPD patients. Eur Respir J 1994;7(10):1871-1876.

7. Ambrosino N, Opasich C, Crotti P, Cobelli F, Tavazzi L, Rampulla C. Breathing pattern, ventilatory drive and respiratory muscle strength in patients with chronic heart failure. Eur Respir J 1994;7(1):17-22.

8. Polkey MI, Green M, Moxham J. Measurement of respiratory muscle strength. Thorax 1995;50(11):1131-1135.

9. Larson JL, Covey MK, Vitalo CA, Alex CG, Patel M, Kim MJ. Maximal inspiratory pressure. Learning effect and test-retest reliability in patients with chronic obstructive pulmonary disease. Chest 1993;104(2):448-453
10. Wilson SH, Cooke NT, Edwards RH, Spiro SG. Predicted normal values for maximal respiratory pressures in caucasian adults and children. Thorax 1984;39(7):535-538.

11. Black LF, Hyatt RE. Maximal respiratory pressures: normal values and relationship to age and sex. Am Rev Respir Dis 1969;99(5):696-702

12. Bruschi C, Cerveri I, Zoia MC, Fanfulla F, Fiorentini M, Casali L, et al. Reference values of maximal respiratory mouth pressures: a population-based study. Am Rev Respir Dis 1992;146(3):790-793.

13. Evans JA, Whitelaw WA. The assessment of maximal respiratory mouth pressures in adults. Respir Care 2009;54(10):1348-1359.

14. Hamnegard CH, Wragg S, Kyroussis D, Aquilina R, Moxham J, Green M. Portable measurement of maximum mouth pressures. Eur Respir J 1994;7(2):398-401.

15. Smith PE, Royall RA. A portable mouth pressure meter. Anaesthesia 1992;47(2):144-145.

16. Klefbeck B, Hamrah NJ. Effect of inspiratory muscle training in patients with multiple sclerosis. Arch Phys Med Rehabil 2003;84(7):994-999.

17. Soudon P, Steens M, Toussaint M. A comparison of invasive versus noninvasive full-time mechanical ventilation in Duchenne muscular dystrophy. Chron Respir Dis 2008;5(2):87-93.

18. Smeltzer SC, Lavietes MH. Reliability of maximal respiratory pressures in multiple sclerosis. Chest 1999;115(6):1546-1552.

19. Truwit JD, Marini JJ. Validation of a technique to assess maximal inspiratory pressure in poorly cooperative patients. Chest 1992; 102(4):1216-1219.

20. Romer LM, McConnell AK. Inter-test reliability for non-invasive measures of respiratory muscle function in healthy humans. Eur J Appl Physiol 2004;91(2-3):167-176.

21. Maillard JO, Burdet L, van Melle G, Fitting JW. Reproducibility of twitch mouth pressure, sniff nasal inspiratory pressure, and maximal inspiratory pressure. Eur Respir J 1998;11(4):901-905.

22. Portney LG, Watkins MP. foundations of clinical research; applications to practice. New Jersey: Pearson Prentice Hall; 2009.

23. Wen AS, Woo MS, Keens TG. How many maneuvers are required to measure maximal inspiratory pressure accurately. Chest 1997; 111(3):802-807.

24. Volianitis S, McConnell AK, Jones DA. Assessment of maximum inspiratory pressure: prior submaximal respiratory muscle activity (warmup) enhances maximum inspiratory activity and attenuates the learning effect of repeated measurement. Respiration 2001;68(1):22-27.

25. Richman J, Makrides L, Prince B. Research methodology and applied statistics; part 3: measurement procedures in research. Physiother Can 1980;32(4):253-257.

26. Shoukri MM, Asyali MH, Donner A. Sample size requirements for the design of reliability study: review and new results. Stat Meth Med Res 2004;13(4):251-271.

27. Bland JM, Altman DG. Statistical methods for assessing agreement between two methods of clinical measurement. Lancet 1986;1(8476): 307-310.

28. Bravo G, Potvin L. Estimating the reliability of continuous measures with Cronbach's alpha or the intraclass correlation coefficient: toward the integration of two traditions. J Clin Epidemiol 1991;44(45):381-390.

29. Hawkes EZ, Nowicky AV, McConnell AK. Diaphragm and intercostal surface EMG and muscle performance after acute inspiratory muscle loading. Respir Physiol Neurobiol 2007;155(3):213-219.

This article is approved for Continuing Respiratory Care Education credit. For information and to obtain your CRCE

(free to AARC members) visit www.RCJournal.com

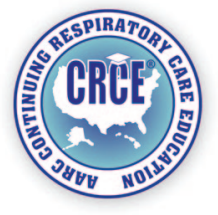

